\title{
Building Bridges - Organelle Tethering Complexes in Subcellular Communication
}

\author{
Markus Islinger ${ }^{1 *}$ and Michael Schrader ${ }^{2}$ \\ ${ }^{1}$ Neuroanatomy, Center for Biomedicine and Medical Technology Mannheim, University of Heidelberg, 68167 Mannheim, Germany \\ ${ }^{2}$ College of Life and Environmental Sciences, Biosciences, University of Exeter, EX4 4QD Exeter, Devon, UK
}

\section{Commentary}

Membrane-bound organelles generate subcellular compartments in order to facilitate individual metabolic pathways which require distinct biochemical environments. In this classical view, organelles have been associated to specific functions such as ATP-generation, lipid- or amino acid metabolism. Quite frequently metabolic pathways are only partially completed in one organelle and intermediate compounds have to be transferred from one organelle to the other. Examples for such pathways are etherlipid synthesis, which is performed in peroxisomes and the endoplasmic reticulum (ER), or cholesterol biosynthesis, which distributes among ER, mitochondria and partially peroxisomes [1]. Moreover, semiautonomous organelles such as mitochondria and peroxisomes are able to grow and multiply [2]. For this reason, they have to incorporate phospholipids, which are predominately synthesized by the ER, into their membranes. Theoretically, such metabolites could be transferred from one organelle to the other by mere diffusion or using cytosolic protein transporters. Remarkably, early ultrastructural studies revealed that organelles frequently show spatial proximities with electron dense material found between the apposed membranes, as observed e.g. for peroxisomes or mitochondria and the smooth ER [35]. Thus, a transfer of metabolites between such regions of membrane apposition would offer a more direct and rapid exchange route. 25 years ago, Jean Vance was for the first time able to purify a specialized ER fraction which comprised a contact site between mitochondria and the ER and was thus named "mitochondria-asssociated membrane of the ER" or MAM [6]. Initial characterization of the MAM's functional properties revealed that the MAM is a site of phospholipid transfer between both organelles. To date the MAM still remains the best characterized organelle contact site. It has not only been associated with the transfer of phosphatidylserine but an increasing number of specific functions such as calcium signaling, mitochondrial fission, mitophagy, ER-stress response, regulation of apoptosis and inflammatory/antiviral responses[7-9]. At the molecular level the MAM exhibits a distinct protein composition, which is characterized by enrichment of a distinct set of ER proteins and their mitochondrial counterparts. According to the plethora of functions mentioned above, it is not clear if there is one single MAM or several individual ER-mitochondria contact sites which are involved in one or some of the specific functions described above[9,10]. With regard to the functional properties of the MAM it is becoming increasingly evident, that organelle contact sites are not only merely locations of rapid metabolite transfer but are involved in important cellular signaling processes. As an example, the MAM appears to regulate mitochondrial ATP synthesis by transmission of $\mathrm{Ca}^{2+}$ ions, which enhance enzymes of the mitochondrial Krebs cycle and ultimately elevate ADP phosphorylation rates [10]. In this respect, ER-mitochondria contact sites have been reported to increase under ER-stress, thereby facilitating ER to mitochondria $\mathrm{Ca}^{2+}$ transfer in order to increase ATP production for the energydemanding ER unfolded protein response [11]. However, if cellular homeostasis cannot be restored, lethal signaling pathways appear to be activated finally triggering controlled cell death. Indeed, an increase in ER-mitochondria contact sites under prolonged ER stress has been described to cause mitochondrial $\mathrm{Ca}^{2+}$ overload and to activate the mitochondrial apoptosis pathway [12]. As depicted in this example, molecule exchange between two subcellular compartments can be readily exploited to regulate complex cellular functions and ultimately influence the decision between cell death and survival. Thus, direct organelle contact sites between further organelles may be similarly integrated into complex cellular regulation circuits and have important physiological functions.

In line with such an assumption, an increasing number of organelle contact sites have been described during recent years [13]: Similar to the mitochondrial contacts, the ER exhibits specialized cellular subcompartments in close apposition to the plasma membrane designated PAM, which are functionally involved in the exchange of membrane lipids and $\mathrm{Ca}^{2+}$ signaling [14]. Likewise, peroxisomes show significant areas of ER apposition, and ER tubules can even be co-purified attached to isolated peroxisomes [15]. In contrast to the MAM and the PAM, the peroxisome-associated membrane of the ER (POAM) contacts remain uncharacterized at the molecular and functional level [13]. Since peroxisomes share metabolic pathways with the ER and have been described to receive phospholipids and even membrane proteins from the ER $[1,16,17]$, the membrane appositions may be involved in such functions. Recently, connections between lysosomes and peroxisomes have also been described and were reported to facilitate the transport of cholesterol from the former to the latter [18]. Accordingly, extracellular cholesterol from low density lipoproteins (LDL) may be transported from the lysosomes, as their primary destination, to peroxisomes where they may be used for the production of bile acids or incorporated into lipid rafts at the peroxisomal membrane. Mitochondria and peroxisomes share an intricate relationship and cooperate in a significant number of metabolic functions such as fatty acid degradation or maintenance of cellular ROS homeostasis [19]. Both organelles also share a significant part of their division machinery, which may mirror the demand for a coordinated biogenesis under distinct metabolic conditions [20]. Division proteins shared by peroxisomes and mitochondria include the dynamin-like fission GTPase DLP1/Drp1, and the membrane adaptor proteins Fis1 (Fission factor 1) and Mff (Mitochondrial fission factor), which can recruit DLP1 to the organelle membranes. Mutations in DLP1 and Mff have been linked to a novel group of disorders with altered mitochondrial and peroxisomal morphology and membrane

*Corresponding author: Markus Islinger, Neuroanatomy, Center for Biomedicine and Medical Technology Mannheim, Medical Faculty Mannheim, University of Heidelberg. 68167 Mannheim, Germany; E-mail: markus.islinger@medma.uni-heidelberg.de

Received: November 09, 2015; Accepted: November 23, 2015; Published November 25,2015

Citation: Islinger M, Schrader M (2015) Building Bridges - Organelle Tethering Complexes in Subcellular Communication. Biochem Anal Biochem 4: 225 doi:10.4172/2161-1009.1000225

Copyright: $\odot 2015$ Islinger M, et al. This is an open-access article distributed under the terms of the Creative Commons Attribution License, which permits unrestricted use, distribution, and reproduction in any medium, provided the original author and source are credited. 
dynamics $[19,21,22]$. Whereas the functional connection between both organelles is undoubtable, our knowledge on physical contacts remains still fragmentary. In mammals appositions between both organelles have been described in ultrastructural studies and mitochondria partially co-purify with peroxisomes as a distinct band in density gradients $[23,24]$. Molecular details of such a connection, however, have only been described in yeast, where a tether between the peroxin Pex11 and the mitochondrial ERMES complex has been recently identified [25].

As described above, functions of organellar contacts span from local metabolite exchange to contribution in cellular signaling pathways. In the latter respect, they have been suggested to act as signaling hubs which bring more than two organelles in close proximity, in order to allow fine-tuning of signals to the specific needs of the cell. Mitochondria and peroxisomes were reported to both contain the mitochondrial viral signaling protein (MAVS), which is involved in the regulation of innate antiviral defense responses [26]. MAVS are membrane receptors for the retinoic acid-inducible genel protein (RIG-I) and transmit signals for the interferon-controlled antiviral response. Recently, MAVS have been additionally localized at the MAM as a part of the ER [27]. Interestingly, the authors reported that MAVS were enriched at junctions between all three organelles, where they could potentially integrate activating and repressing signals transmitted by the individual organelles. Another example for a triple contact between those three organelles was reported from S. cerevisiae [28]. Peroxisomes were additionally shown to significantly juxtapose with specialized mitochondrial regions enriched in the pyruvate dehydrogenase complex. Consequently, the functional significance of these triple organelle hubs may be associated with the coordination of cellular energy production according to the demands for anabolic processes of the cell. As illustrated by these examples, complex organelle contact sites appear to form to connect isolated entities of interactive regulation networks in order to flexibly fine-tune cellular homeostasis.

In order to stabilize local membrane appositions but also permit dynamic alterations in connectivity, opposing organelles have to be mechanically interconnected by specialized membrane structures. Such tethering complexes might consist of dimers between two membrane proteins at the opposing organelle membranes, but may include larger protein complexes or protein/membrane lipid interactions [13]. Numerically, the most prominent group comprises the dimeric tethers, which are often formed between two adjacent membrane proteins. Examples for such complexes include the MAM-mitochondria tethers between Bap31 and Fis1, Mfn1 and Mfn2 as well as VAPB and PTPIP51. Extended synaptotagmins (E-Syt) and junctophilins are PAM constituents interacting with phosphatidylinositolphosphate (PIP) lipids at the cytosolic side of the plasma membrane; likewise synaptotagmin 7 on lysosomes has been described to tether to PI $[4,5]$ $\mathrm{P}_{2}$ in the peroxisomal membrane. A prominent example for a larger protein association is the "ER-mitochondria encounter structure" (ERMES) connecting MAM and mitochondria in yeast [29]. The ERMES complex consists of 5 core proteins: $\mathrm{Mdm} 10$ and $\mathrm{Mdm} 34$ at the outer mitochondrial membrane, cytosolic Mdm 12 and two Mmm1 molecules spanning the ER membrane. In mammals the ERMES complex is not conserved, however, other oligomeric tethering complexes have been described. $\mathrm{Ca}^{2+}$ exchange between ER and mitochondria is facilitated by inositol 1,4,5-triphosphate receptors (IP3R) and voltage-dependent anion channel (VDAC), which are connected by cytosolic GRP75 chaperones [30]. Remarkably, a disruption of this complex reduced insulin-induced signaling in mammalian RNAi-based cell culture systems and knockout mouse models [31]. Thus, the organelle contact sites represented by the MAM-associated protein complexes appear to be new players in the development of diabetic insulin resistance. At the molecular level, the tethering complexes described above can likely be regarded as cores of larger protein assemblies, which allow close regulation of the abundance and temporal stability of individual organellar contact sites in order to react to the changing physiological requirements of a cell. In line with such an assumption, additional interaction partners of the ERMES complex or the IP3R-VDAC tether have already been described [32,33]. Now, 25 years after the initial biochemical characterization of the MAM as a specialized contact site between mitochondria and the ER, our knowledge on functionality and molecular components of individual organellar contacts has increased tremendously. Nevertheless, we are only beginning to understand the complexity of interorganelle connections and the signaling pathways integrated in such systems. New findings, as exemplified by the discoveries on the impact of the MAM integrity in insulin resistance, imply that organelle interaction sites may play a significant and previously neglected role in the development of human diseases and merit future investigation.

\section{Acknowledgements}

We would like to thank $\mathrm{J}$. Costello for comments on the manuscript. This work was supported by BBSRC (BB/K006231/1) and FP-7-PEOPLE-2012-Marie CurieITN 316723 PERFUME (to M.S.)

\section{References}

1. Schrader M Grille S, Fahimi HD, Islinger M (2013) Peroxisome interactions and cross-talk with other subcellular compartments in animal cells. Subcell Biochem 69: 1-22.

2. Schrader M, Yoon Y (2007) Mitochondria and peroxisomes: are the 'big brother' and the 'little sister' closer than assumed? BioEssays : news and reviews in molecular, cellular and developmental biology 29: 1105-1114.

3. Novikoff A, Shin W-Y (1964) The endoplasmic reticulum in the Golgi zone and its relations to microbodies, Golgi apparatus and autophagic vacuoles in rat liver cells. J Microsc 3: 187-206.

4. Morre DJ, Merritt WD, Lembi CA (1971) Connections between mitochondria and endoplasmic reticulum in rat liver and onion stem. Protoplasma 73: 43-49.

5. Copeland DE, Dalton AJ (1959) An association between mitochondria and the endoplasmic reticulum in cells of the pseudobranch gland of a teleost. $J$ Biophys Biochem Cytol 5: 393-396.

6. Vance JE (1990) Phospholipid synthesis in a membrane fraction associated with mitochondria. J Biol Chem 265: 7248-7256.

7. Raturi A; Simmen T (2013) Where the endoplasmic reticulum and the mitochondrion tie the knot: the mitochondria-associated membrane (MAM). Biochim Biophys Acta 1833: 213-224.

8. Marchi S, Patergnani S, Pinton P (2014) The endoplasmic reticulummitochondria connection: one touch, multiple functions. Biochim Biophys Acta 1837: $461-469$

9. Vance JE (2014) MAM (mitochondria-associated membranes) in mammalian cells: lipids and beyond. Biochim Biophys Acta 1841(4): 595-609.

10. Rowland AA, Voeltz GK (2012) Endoplasmic reticulum-mitochondria contacts: function of the junction. Nat Rev Mol Cell Biol 13: 607-625.

11. Bravo R, Gutierrez T, Paredes F, Gatica D, Rodriguez AE, et al. (2012) Endoplasmic reticulum: ER stress regulates mitochondrial bioenergetics. Int $J$ Biochem Cell Biol 44: 16-20.

12. Chami M, Oulès B, Szabadkai G, Tacine R, Rizzuto R, et al. (2008) Role of SERCA1 truncated isoform in the proapoptotic calcium transfer from ER to mitochondria during ER stress. Molecular cell. 32: 641-651.

13. Schrader M, Godinho LF, Costello JL, Islinger M (2015) The different facets of organelle interplay-an overview of organelle interactions. Front Cell Dev Biol 3: 56

14. Stefan CJ, Manford AG, Emr SD (2013) ER-PM connections: sites of information transfer and inter-organelle communication. Curr Opin Cell Biol 25: 434-442.

15. Zaar K, Völkı A, Fahimi HD (1987) Association of isolated bovine kidney cortex peroxisomes with endoplasmic reticulum. Biochim Biophys Acta 897: 135-142. 
Citation: Islinger M, Schrader M (2015) Building Bridges - Organelle Tethering Complexes in Subcellular Communication. Biochem Anal Biochem 4: 225. doi:10.4172/2161-1009.1000225

Page 3 of 3

16. Raychaudhuri S, Prinz WA (2008) Nonvesicular phospholipid transfer between peroxisomes and the endoplasmic reticulum. Proc Natl Acad Sci U S A 105: 15785-15790.

17. van der Zand A Braakman I, Tabak HF (2010) Peroxisomal membrane proteins insert into the endoplasmic reticulum. Mol Biol Cell 21: 2057-2065.

18. Chu BB, Liao YC, Qi W, Xie C, Du X, et al. (2015) Cholesterol Transport through Lysosome-Peroxisome Membrane Contacts. Cell 161: 291-306.

19. Schrader M Costello J, Godinho LF, Islinger M (2015) Peroxisome-mitochondria interplay and disease. J Inherit Metab Dis 38: 681-702.

20. Schrader M, Costello JL, Godinho LF, Azadi AS, Islinger M (2015) Proliferation and fission of peroxisomes - An update. Biochim Biophys Acta.

21. Waterham HR Koster J, van Roermund CW, Mooyer PA, Wanders RJ, et al (2007) A lethal defect of mitochondrial and peroxisomal fission. N Engl J Med 356: 1736-1741.

22. Shamseldin HE, Alshammari M, Al-Sheddi T, Salih MA, Alkhalidi H, et al. (2012) Genomic analysis of mitochondrial diseases in a consanguineous population reveals novel candidate disease genes. J Med Genet 49: 234-241.

23. Hicks L, Fahimi HD (1977) Peroxisomes (microbodies) in the myocardium of rodents and primates. A comparative Ultrastructural cytochemical study. Cell Tissue Res 175: 467-481.

24. Islinger M, Luers GH, Zischka H, Ueffing M, Volkl A (2006) Insights into the membrane proteome of rat liver peroxisomes: microsomal glutathione-Stransferase is shared by both subcellular compartments. Proteomics 6: 804-816.

25. Mattiazzi Usaj M, Brložnik M, Kaferle P, Žitnik M, Wolinski H, et al. (2015) Genome-Wide Localization Study of Yeast Pex11 Identifies Peroxisome-
Mitochondria Interactions through the ERMES Complex. J Mol Biol 427: 2072 2087

26. Dixit E Boulant S, Zhang Y, Lee AS, Odendall C, et al. (2010) Peroxisomes are signaling platforms for antiviral innate immunity. Cell 141: 668-681.

27. Horner SM, Liu HM, Park HS, Briley J, Gale M Jr (2011) Mitochondrialassociated endoplasmic reticulum membranes (MAM) form innate immune synapses and are targeted by hepatitis C virus. Proc Natl Acad Sci U S A 108 14590-14595.

28. Cohen Y, Klug YA, Dimitrov L, Erez Z, Chuartzman SG, et al. (2014) Peroxisomes are juxtaposed to strategic sites on mitochondria. Molecular bioSystems 10: 1742-1748.

29. Lang A John Peter AT Kornmann B2 (2015) ER-mitochondria contact sites in yeast: beyond the myths of ERMES. Curr Opin Cell Biol 35: 7-12.

30. Szabadkai G Bianchi K, Várnai P, De Stefani D, Wieckowski MR, et al. (2006) Chaperone-mediated coupling of endoplasmic reticulum and mitochondrial Ca2+ channels. J Cell Biol 175: 901-911.

31. Tubbs E, Theurey P, Vial G, Bendridi N, Bravard A, et al. (2014) Mitochondriaassociated endoplasmic reticulum membrane (MAM) integrity is required for insulin signaling and is implicated in hepatic insulin resistance. Diabetes 63: 3279-3294.

32. Kornmann B, Osman C, Walter P (2011) The conserved GTPase Gem1 regulates endoplasmic reticulum-mitochondria connections. Proc Natl Acad Sci U S A 108: 14151-14156.

33. Paillard M, Tubbs E, Thiebaut PA, Gomez L, Fauconnier J, et al. (2013) Depressing mitochondria-reticulum interactions protects cardiomyocytes from lethal hypoxia-reoxygenation injury. Circulation 128: 1555-1565. 